\title{
DIRECTION-ADAPTIVE GREY-LEVEL MORPHOLOGY. APPLICATION TO 3D VASCULAR BRAIN IMAGING
}

\author{
Olena Tankyevych ${ }^{1}$, Hugues Talbot ${ }^{1}$, Petr Dokladál ${ }^{2}$, Nicolas Passat ${ }^{3}$ \\ ${ }^{1}$ Université Paris-Est, Laboratoire informatique Institut Gaspard-Monge, \\ Unité mixte de recherche ESIEE/UMLV/CNRS, Noisy-le-Grand, France \\ ${ }^{2}$ Centre de Morphologie Mathématique \\ Ecole Nationale Supérieure des Mines de Paris, Fontainebleau, France \\ ${ }^{3}$ Université de Strasbourg, LSIIT UMR 7005, Strasbourg, France
}

\begin{abstract}
Segmentation and analysis of blood vessels is an important issue in medical imaging. In 3D cerebral angiographic data, the vascular signal is however hard to accurately detect and can, in particular, be disconnected. In this article, we present a procedure utilising both linear and morphological methods for blood vessel edge enhancement and reconnection. More specifically, multi-scale second-order derivative analysis is performed to detect candidate vessels as well as their orientation. This information is then fed to a spatially-variant morphological filter for reconnection and reconstruction. The result is a fast and effective method.
\end{abstract}

Index Terms - Adaptive morphology, spatially-variant morphology, vascular imaging, vesselness, Hessian-based filtering, directional filtering.

\section{INTRODUCTION}

Vascular network detection from angiographic data is an important issue, justified by various applications (pathology detection, surgical planning, etc.). In this context, 3D Computed Tomography Angiography (CTA) provides a well-detailed visualisation of vascular networks until the resolution of the generated images (i.e. $0.5 \mathrm{~mm}$ ). However, the small size and the complexity of the vascular structures, coupled to noise, acquisition artifacts, and blood signal heterogenity (especially signal discontinuity) make the analysis of such data a challenging task, thus justifying intensive efforts devoted to segmentation purpose.

There exist a multitude of works on vessel segmentation (see [1] for a survey), but only very few are capable of segmenting the smallest tubular objects. Among them, the second-order derivative based methods that rely on vessel geometrical models $[2,3,4]$ can perform in a multiscale fashion, thus detecting different sized objects. However, in practice, such vesselness filters can segment large objects and are more likely to ignore the smallest ones.
Consequently, specifically-designed filters must be considered to filter thin elongated objects. For such structures, it is assumed that one dimension is still long, everywhere tangent to the object. Filtering can thus be applied along this direction without necessarily altering the object structure. In morphology, one can use families of segments as structuring elements, selecting the one that best matches the objects everywhere [5]. This, however, requires directional sampling, which may be prohibitive in 3D.

Recently, two similar 2D approaches were proposed combining the analysis of orientation from either the Hessian or the structure tensor, and using spatially-variant morphology to bridge the disconnection gaps [6,7]. Spatially-variant morphology has also been used for adaptive filtering on grey-level and colour images in [8]. This is a productive approach, as long as the resulting filter is indeed a morphological filter, and directions are well estimated.

This article is a 3D extension of our previous work [7] on a 2D morpho-Hessian filter. Here, we especially focus on cerebral angiographic image segmentation. Our final purpose is in particular to segment vessels to further perform vein/artery classification. As a primary step, we aim at filtering and segmenting vessels, while detecting the smaller ones and correctly reconnecting them to larger vessels.

The procedure begins with a vesselness-based multiscale segmentation similar to the one proposed in [2]. Then, based on the detected principal vessel directions, a morphological closure is carried out on the considered image. In the last step, the vascular network is reconstructed based on the vesselnessdetected objects propagating into the morphologically filtered image. The results of the filtering are compared to the vessel enhancing diffusion filter by Manniesing [9]; they emphasise the detection and reconnection of the smaller vessels.

\section{METHODS}

The proposed procedure consists of the following three steps: 1) detection of vessel-like objects and of their directions by 
using a multi-scale vesselness method, 2) propagation of intensities inside the vessel and reconnection using a morphological closing by a spatially-variant structuring element according to the detected directions, and 3) reconstruction of vascular tree from the vesselness detected objects into the morphologically closed image. These steps are detailed below.

\subsection{Tubular object detection}

The first step of the vascular image filtering is the segmentation of the tubular structures. This is done with the Hessianbased filtering measure. The Hessian matrix is obtained from the Gaussian second derivative analysis of the 3D image $F$ at each voxel in its six principal directions:

$$
\mathbf{H}=\left[\begin{array}{ccc}
F_{x x} & F_{x y} & F_{x z} \\
F_{y x} & F_{y y} & F_{y z} \\
F_{z x} & F_{z y} & F_{z z}
\end{array}\right]
$$

By the principal value analysis, the Hessian matrix $\mathbf{H}$ can be decomposed into three principal values, $\lambda_{1}, \lambda_{2}$ and $\lambda_{3}$ ( $\left.\left|\lambda_{1}\right| \leq\left|\lambda_{2}\right| \leq\left|\lambda_{3}\right|\right)$ and $\mathbf{e}_{\mathbf{1}}$, $\mathbf{e}_{2}$ and $\mathbf{e}_{\mathbf{3}}$ their corresponding eigenvectors, respectively. When $\lambda_{1}$ is close to zero and much smaller than $\lambda_{2}$ and $\lambda_{3}$, the principal direction of the (bright) vessel is given by $\mathbf{e}_{\mathbf{1}}$. Among the most popular definitions of vessel-like object measures [2, 4, 3], we have chosen the vesselness function provided in [2]. Indeed, this function, formulated as follows, seems to be advantageous due to its multiscale representation:

$\nu(x, \sigma)=\left\{\begin{array}{l}0 \text { if } \lambda_{2}>0 \text { or } \lambda_{3}>0, \\ \left(1-e^{\frac{-R_{A}^{2}}{2 \alpha^{2}}}\right) \cdot e^{-\frac{R_{B}^{2}}{2 \beta^{2}}} \cdot\left(1-e^{\frac{-S^{2}}{2 \gamma^{2}}}\right) \text { otherwise }\end{array}\right.$

with

$$
\begin{aligned}
& R_{A}=\frac{\left|\lambda_{2}\right|}{\left|\lambda_{3}\right|}, \\
& R_{B}=\frac{\left|\lambda_{1}\right|}{\sqrt{\left|\lambda_{2} \lambda_{3}\right|}}, \\
& S=\left\|H_{\sigma}\right\|=\sqrt{\sum_{j} \lambda_{j}^{2}},
\end{aligned}
$$

in which $R_{A}$ differentiates between plate and line like objects, $R_{B}$ describes blob-like ones, and $S$ accounts for the intensity difference between objects (vessels) and background. Parameters $\alpha, \beta$ and $\gamma$ influence the weight of the according objects. The final vesselness result is produced by its best response at different scales $\sigma$ for each voxel $x$.

\subsection{Direction-adaptive morphological filter}

Let $\mathcal{L}$ be a family of functions, or images, defined as a mapping from the support $D$ to the set of values $V$.

Let $\delta, \varepsilon: \mathcal{L} \rightarrow \mathcal{L}$ be a dilation and an erosion of $f \in \mathcal{L}$ given by

$$
\begin{aligned}
& {\left[\delta_{B}(f)\right](x)=\left[\bigvee_{b \in B(x)} f_{b}\right](x)} \\
& {\left[\varepsilon_{B}(f)\right](x)=\left[\bigwedge_{b \in \widehat{B}(x)} f_{b}\right](x)}
\end{aligned}
$$

where $f_{b}$ denotes the translation of $f$ by $b$ computed as $f_{b}(x)$ $=f(x-b)$. The set $B$ is the structuring element. Usually, $B$ is translation invariant, defined as $B \subset D$.

However, in the context of spatially variant morphology, $B$ is no longer a set but a family of sets (we then say that $B$ is a structuring function). This structuring function $B$ is then defined as $B: D \rightarrow \mathcal{P}(D)$ (where $\mathcal{P}(D)=\{X \mid X \subseteq$ $D\}$ ). Hence, $B(x)$ denotes the structuring element $B$ used at location $x$ by

The set $\widehat{B}$, used in Eq. 5, called transpose of $B$, is defined

$$
\widehat{B}(x)=\{y \mid x \in B(y)\}
$$

Notice that, contrary to the invariant transposition $\widehat{B}=\{x \mid$ $-x \in B\}$, which is a set reflection, the spatially-variant transposition Eq. 6 is non local and requires an exhaustive search. Such a search is prohibitive for composing filters based on series of dilation and erosion, to form a closing or an opening

$$
\varphi_{B} f=\varepsilon_{B}\left(\delta_{B}(f)\right) ; \gamma_{B} f=\delta_{B}\left(\varepsilon_{B}(f)\right)
$$

These operations, implemented by using the definitions Eqs. 4,5 , both reauire $B$ and $\widehat{B}$. Nevertheless, the transposition of $B$ rapidly becomes costly for larger structuring elements, and particularly in 3D.

\subsubsection{Implementation}

To compute a spatially variant closing $\varphi_{B} f(x)$ using Eq. 7, one needs to compute the erosion of dilation of $f(x)$. The inf/sup-of-functions based definitions Eqs. 4, 5 are usually computed sequentially in the raster scan order according to the following scheme.

$$
\begin{array}{r}
{\left[\delta_{B}(f)\right](x)=\max _{b \in B(x)} f(x-b)} \\
{\left[\varepsilon_{B}(f)\right](x)=\min _{b \in \widehat{B}(x)} f(x-b)=\min _{\{y \mid x \in B(y)\}} f(x-y)}
\end{array}
$$

The erosion (Eq. 8) is computed in $\mathcal{O}(|B(x)| .|D|)$. In the following erosion (Eq. 9) given some $x$, the set $\{y \mid x \in B(y)\}$ is unknown and is then computed by exhaustive search in $\mathcal{O}\left(|D|^{2}\right)$. However, relaxing the sequential order of computing, the erosion can be computed more efficiently. Instead of computing $\varepsilon_{B}(f)$ sequentially for all $x$, one can sequentially read the input $f$ and update $\varepsilon_{B}(f)$ at all locations of $B(x)$, where $x$ contributes. 


\subsection{Algorithm}

The Hessian matrix in its eigen form is produced for each voxel on multiple scales of the image. The choice and number of scales is chosen in order to detect vessels with different radii. The vesselness value is calculated across these scales according to Eq. 2, where the maximum response is chosen at the end for each voxel bringing along the principal direction obtained with the Hessian matrix.

In the second step of the filtering, the morphological closing operation is performed over the image with the aim of reconnecting vessels. In order to propagate objects in the space, it is necessary to have a dense direction field. In other diffusion methods $[9,6]$, a directional tensor was computed using an average squared gradient, then expanded using gradient vector flow, in order to propagate the direction field everywhere. In our case, this is not necessary since we only need the directional information as far as the dilation can reach. As the image is processed with a spatially varying structuring element whose direction is obtained according to the principal voxel direction according to the vesselness filter, we perform the dilation first. At first, only the directional information is propagated in the direction indicated by the Hessian. Then, with the second dilation operation, the actual intensity image is dilated according to the directions obtained at the previous dilation. After follows the adjunct erosion operation as described in section 2.2.1. This ensures an idempotent result, which guarantees that the resulting filter obeys all morphological rules.

\subsection{Morphological reconstruction}

Following the morpho-Hessian closing - which performs like an edge-enhancing inverse diffusion filter in some ways noise levels are generally increased. As our aim is mostly vessel reconnection, we perform grey-level reconstruction [10] using the initial vesselness image as marker, and the result of the morphological closing as mask. This readily eliminates all non-connected noise.

\section{RESULTS AND DISCUSSION}

The filters were written with the ITK library using the available implementations of vesselness and vessel enhancement diffusion functions.

For experiments of the filtering method, the considered vesselness parameters have been set similarly to [9]: $\alpha=\beta$ $=0.5$ and $\gamma=100$, in order to avoid any bias when comparing the proposed method with the vesselness diffusion method proposed by Manniesing et al. [9].

In general, the lower scales are more sensitive to noise. Moreover, in [11] it has been reported that the vesselness response curve differs not more than 3 percent between $\sigma=1$ and $\sigma=2 \sqrt{2}$ using a set of discrete values

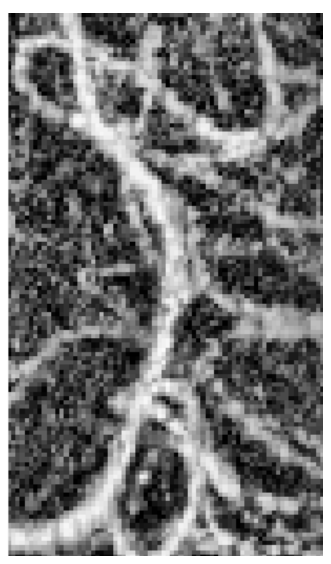

(a)

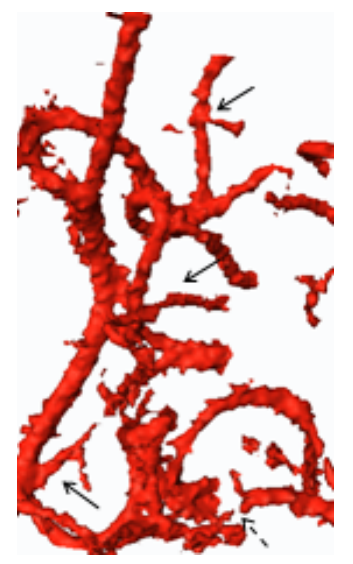

(c)

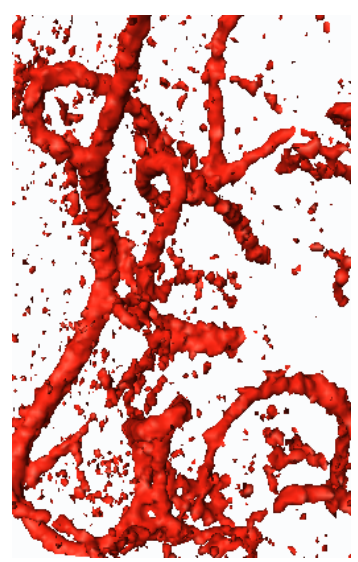

(b)

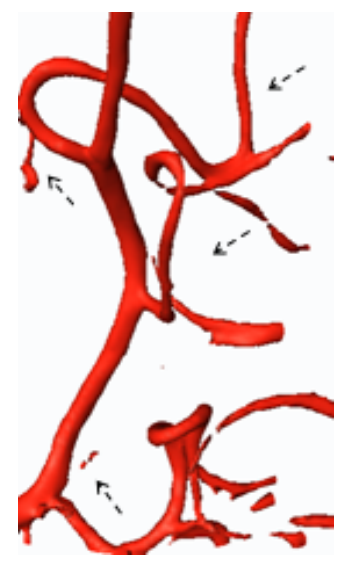

(d)
Fig. 1. Filtering example on a low-dose CT angiography image. (a) Original image maximal intensity projection, (b) original image volume rendering, (c) morpho-hessian surface rendering, (d) vesselness surface rendering. Plain arrows indicate areas of reconnection. Dashed arrows point to missed out vessels.

$\sigma_{i} \in 1, \sqrt{2}, 2,2 \sqrt{2}$, which is applicable for most applications. Therefore, for our filtering method, we used the scales between $\sigma=1$ and $\sigma=2 \sqrt{2}$, but with three logarithmic natural incrementation steps, as noted in [12] to be valid mainly for coarse scales.

Segmentation excerpt of a CTA data is illustrated in Figure 1. The original and filtered images are surface-rendered with the same threshold. The surface-rendered image (Figure 1a) and its projection (Figure 1b), show that it is densely disrupted by noise. In Figure 1d, it can be observed that the vesselness diffusion filters out the noise while smoothing the surface of vessels. However, doing so, it also removes the smaller tubular objects. In the contrast, morpho-Hessian filter (Figure 1c) keeps the same rough vessel surface as the original surface rendering, but it also preserves and reconnects the smaller objects and eliminates the noise (the plain arrows in 
the images indicate the places where the filter has managed to reconstruct or/and reconnect vessels; the dashed arrows indicate the places that should have been reconstructed in a better fashion). It can be noticed that some reconnections are much thinner than the vessel parts. This is due to the size of our structuring element, which is a path of one pixel thickness.

Even though the minimal used scale $(\sigma=1)$ should not be sufficient to detect the smallest vessel in the CT image $(0.5 \mathrm{~mm})$, the fact that the final morpho-Hessian image is reconstructed from the vesselness result provides us with reconnections of smaller arteries with larger vessels by propagating intensities in the direction of the later.

\section{CONCLUSION}

In this article, we have presented a combined linear-morphological filter for detecting, edge-enhancing and reconnecting blood vessels. The results obtained by applying this filter on real 3D CTA data, compared to those obtained from a vessel enhancing diffusion filter [9], emphasise the ability of the proposed approach to reconnect the smaller parts of the vessel network with the larger ones.

It has to be noticed that some parts of the approach could however be improved. First, the filter is based on the directions computed from the Hessian matrix, and then strongly relies on the quality of these computed directions. The direction computation could actually be improved by also considering the first-order derivative of the image (as in [6]).

Second, instead of choosing only one scale (namely the one for which the response is maximal), it could be interesting to consider several potentially relevant scales and to combine the results obtained at each of them in order to increase the robustness of the process. The handling of scales w.r.t. to the topology of the vascular tree could also be considered in further works.

Third, the thin reconnections could be improved using rectangular spatially-variant structuring elements, as proposed in [13], using the eigenvalues of the Hessian matrix to determine their size.

As a result, we have come up with an efficient reconstruction filter that can be of help in segmentation and filtering of thin objects. The main perspective of this work is now to be able to efficiently segment the whole vascular tree and to discriminate arteries from veins.

\section{ACKNOWLEDGMENTS}

We wish to thank the following medical personnel from Colmar Hospital: A. Tournade, M. Musacchio, H. Oesterlé, M. Lagneau, who provided medical expertise and data.

\section{REFERENCES}

[1] C. Kirbas and F. Quek, "A review of vessel extraction techniques and algorithms," ACM Computing Surveys, vol. 36, no. 2, pp. 81-121, 2004.

[2] A.F. Frangi, W.J. Niessen, K.L. Vincken, and M.A. Viergever, "Multiscale vessel enhancement filtering," in MICCAI, 1998, vol. 1496 of LNCS, pp. 130-137.

[3] K. Krissian, G. Malandain, N. Ayache, and Y. Vaillant, R. andTrousset, "Model-based detection of tubular structures in 3D images," Computer Vision and Image Understanding, vol. 80, no. 2, pp. 130-171, 2000.

[4] Y. Sato, S. Nakajima, N. Shiraga, H. Atsumi, S. Yoshida, T. Koller, G. Gerig, and R. Kikinis, "Three-dimensional multi-scale line filter for segmentation and visualization of curvilinear structures in medical images," Medical Image Analysis, vol. 2, no. 2, pp. 143-168, 1998.

[5] P. Soille and H. Talbot, "Directional morphological filtering," IEEE Transactions on Pattern Analysis and Machine Intelligence, vol. 23, no. 11, pp. 1313-1329, 2001.

[6] R. Verdú-Monedero and J. Angulo, "Spatially-variant directional mathematical morphology operators based on a diffused average squared gradient field," in ACIVS. 2008, vol. 5259 of $L N C S$, pp. 542-553, Springer.

[7] O. Tankyevych, H. Talbot, and P. Dokladal, "Curvilinear morpho-Hessian filter," in IEEE ISBI, 2008, pp. 10111014.

[8] R. Lerallut, E. Decencière, and F. Meyer, "Image filtering using morphological amoebas," Image and Vision Computing, vol. 25, no. 4, pp. 395-404, 2007.

[9] R. Manniesing, M.A. Viergever, and W.J. Niessen, "Vessel enhancing diffusion: A scale space representation of vessel structures," Medical Image Analysis, vol. 10, no. 6, pp. 815-825, 2006.

[10] L. Vincent, "Morphological grayscale reconstruction in image analysis: Applications and efficient algorithms," IEEE Transactions on Image Processing, vol. 2, no. 2, pp. 176-201, 1993.

[11] Y. Sato, C.-F. Westin, A. Bhalerao, S. Nakajima, S. Shiraga, S. Tamura, and R. Kikinis, "Tissue classification based on 3D local intensity structures for volume rendering," IEEE Transactions on Visualization and Computer Graphics, vol. 6, no. 2, pp. 160-180, 2000.

[12] J. J. Koenderink, "The structure of images," Biological Cybernetics, vol. 50, no. 5, pp. 363-370, 1984.

[13] P. Dokladal and E. Dokladalova, "Grey-scale morphology with spatially-variant rectangles in linear time," in ACIVS, 2008, vol. 5259 of LNCS, pp. 674-685. 